\section{INSTITUCIONES EDUCATIVAS EN ESCENARIOS INESPERADOS}

\author{
EDUCATIONAL INSTITUTIONS IN UNEXPECTED SETTINGS
}

\author{
Cinthia Vanesa Cossio ${ }^{1}$ - Mirian Anahí Gomez Alegre ${ }^{2}$
}

Fecha de recepción: 08-10-2020

Fecha de aceptación y versión final: 01-06-2021

\section{Resumen}

En el siguiente artículo vamos a analizar el impacto que ha tenido la pandemia del coronavirus (COVID-19) en nuestro país, puntualmente en el ámbito de la educación. Intentaremos realizar una descripción sintética de cómo a partir de la suspensión de las clases presenciales, las instituciones educativas se vieron forzadas a implementar una enseñanza remota de emergencia que potenció el uso de las tecnologías de la información y comunicación (TIC) para sostener la continuidad pedagógica y mantener los vínculos entre docentes y estudiantes. Nuestro principal interés está relacionado con reflexionar acerca de las acciones institucionales y las adaptaciones que se implementaron. A su vez, analizaremos los matices que podrían surgir en algunos escenarios institucionales y trataremos de detectar algunas alternativas que se podrían vislumbrar frente a un escenario de pospandemia, apuntando la mirada hacia el futuro ( $\mathrm{y}$ futuros posibles), preguntándonos sobre la importancia de idear o repensar proyectos institucionales que incluyan las TIC de manera integral y a largo plazo.

Palabras clave: educación- tecnologías de la información y la comunicación- instituciones de enseñanza- educación en pandemia.

\begin{abstract}
In the following article we are going to analyze the impact that the coronavirus pandemic (COVID-19) has had in our country, specifically in the field of education. We will try to make a synthetic description of how, from the suspension of face-to-face classes, educational institutions were forced to implement emergency remote teaching that promoted the use of information and communication technologies (ICT) to sustain pedagogical continuity. and maintain the links between teachers and students. Our main interest is related to reflecting on the institutional actions and adaptations that were implemented. At the same time, we will analyze the nuances that could arise in some institutional settings and we will try to detect some alternatives that could be glimpsed in the face of a post-pandemic scenario, pointing to the

\footnotetext{
${ }^{1}$ Especialista en Tecnología Educativa por la Universidad de Buenos Aires (UBA). Licenciada en Ciencias de la Educación (UBA). Facilitadora Pedagógica Digital en Ministerio de Educación del Gobierno de la Ciudad de Buenos Aires. Profesora consejera en la Universidad Metropolitana para la Educación y el Trabajo (UMET). Docente de la materia Integración Tecnológico. Académica en la Universidad de Ciencias Empresariales y Sociales (UCES virtual). Adscripta de la Cátedra Educación a Distancia y Educación a Distancia y Virtual de la carrera de Licenciatura en Ciencias de la Educación de la Facultad de Filosofía y Letras, UBA. Asesora pedagógica. Correo electrónico: cincossio@gmail.com

${ }^{2}$ Especialista y Maestranda de la Maestría en Tecnología Educativa por la Facultad de Filosofía y Letras de la Universidad de Buenos Aires (UBA). Licenciada en Ciencias de la Educación (UBA). Profesional de gestión interna de la Gerencia de Formación y Capacitación del Instituto Nacional de Tecnología Agropecuaria (INTA). Adscripta de la Cátedra Educación a Distancia y Educación a Distancia y Virtual de la carrera de Licenciatura en Ciencias de la Educación de la Facultad de Filosofía y Letras, UBA. Asesora pedagógica y tutora virtual. Correo electrónico: mirigomezalegre@gmail.com
} 
RIIE (2021), Año 12 (15), 55- 67.

DOI: http://dx.doi.org/10.30972/riie.12155568

future (and possible futures), asking ourselves about the importance of devising or rethinking institutional projects that include ICT in a comprehensive and long-term manner.

Key words: education- information and communication technologies- educational institutionspandemic education. 


\section{Introducción}

Durante los primeros meses del año 2020 nuestro país se ha visto sacudido, en varios y profundos aspectos, por el desarrollo de la pandemia del coronavirus (COVID- 19). Si bien en algunos países del mundo ya circulaba hacia finales del 2019, recién a principios del 2020 se advirtió su expansión mundial con los niveles alarmantes de propagación y gravedad. Particularmente, la Organización Mundial de la Salud (OMS), anunció la caracterización del virus como una pandemia el 11 de marzo del año 2020.

Ante esta situación particular e inesperada, el Estado argentino decidió tomar medidas de aislamiento social, preventivo y obligatorio (ASPO) mediante el DNU 297/2020 (y sus modificatorias subsiguientes), medidas que fueron de vital importancia para hacer frente a la situación epidemiológica en pos de limitar el avance del coronavirus y mitigar el impacto sanitario. Esta decisión, consecuentemente, implicó adecuarse a formas diferentes de funcionamiento: algunas actividades laborales se declararon esenciales -por lo que se desarrollaron de forma presencial 0 semipresencial- y otras se desarrollaron bajo la modalidad de trabajo remoto. En definitiva, este hecho, nos comprometió a repensar nuestras formas de vida y relaciones sostenidas hasta antes del aislamiento.

En este escenario concreto, las instituciones, actores y comunidades educativas se vieron afectadas rápidamente: desde el mes de marzo de 2020 se decretó la suspensión de las clases presenciales en todos los niveles educativos en Argentina (aunque fue una decisión adoptada por la mayor parte de los países del mundo). Como podemos ver en la Figura 1, debido a la suspensión de las clases presenciales en nuestro país, por la pandemia del coronavirus COVID-19, las aulas físicas de todas las instituciones educativas nacionales han quedado vacías.

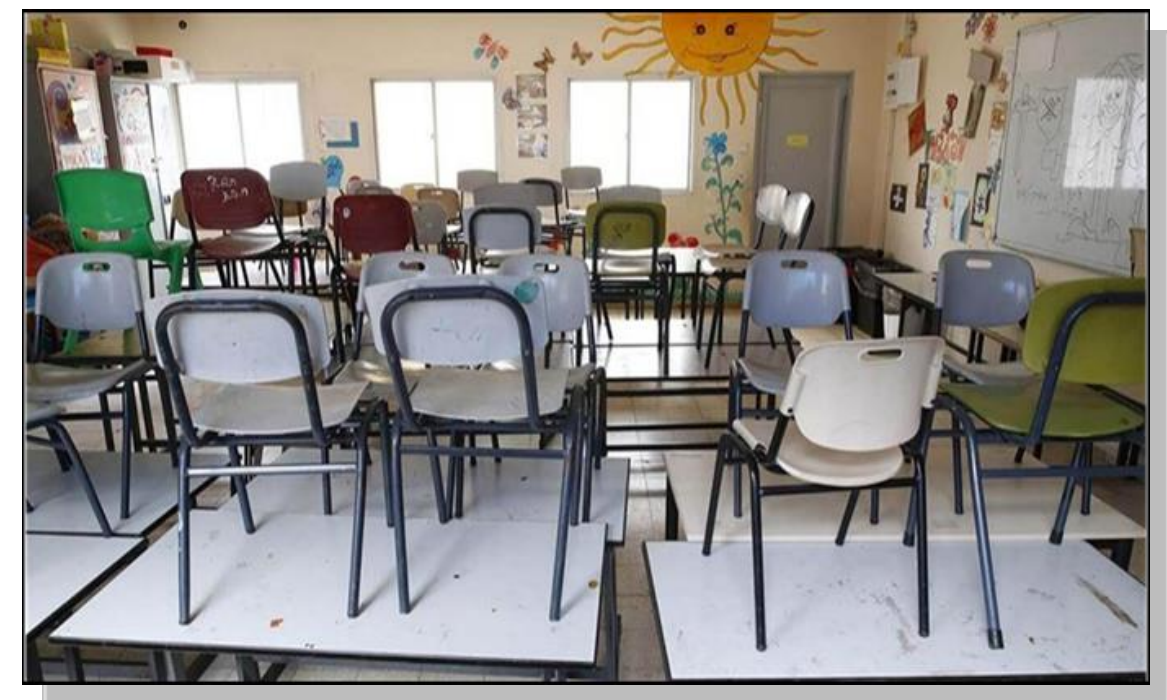

Fuente: "Educación en tiempos de pandemia" (19/03/2020), Universidad Nacional de San Martín (UNSAM). Recuperado de https://www.unsam.edu.ar/tss/educacion-en-tiempos-de-pandemia/

En este contexto de emergencia e incertidumbre, fue necesario repensar de forma abrupta cuestiones que involucran configuraciones institucionales, el trabajo pedagógico, las prácticas de enseñanza y de aprendizaje. La finalidad era tratar de garantizar la continuidad de las trayectorias educativas y sostener el vínculo pedagógico durante el período de suspensión de las clases presenciales. 
Para llevar a cabo acciones que impactaran de forma rápida, consideramos clave el rol del Estado y el despliegue de políticas públicas en el ámbito educativo para establecer consensos respecto de proteger el derecho a la educación, brindando las condiciones que sostengan el vínculo pedagógico y garanticen la continuidad de los aprendizajes de los y las estudiantes. En este sentido podemos mencionar que, todas las provincias de nuestro país cuentan con herramientas digitales educativas, aunque con alcances y recursos dispares (AxE, 2020). En los meses de aislamiento, se observaron acciones que configuraron escenarios alternativos en tiempo récord e inéditos para mediatizar de manera remota el vínculo y el encuentro pedagógico entre docentes y estudiantes, donde las Tecnologías de la Información y la Comunicación (TIC) han sido y están siendo empleadas de manera ampliada.

En esta línea, es posible percibir que si bien apuntan a favorecer el derecho a educar y quizás aspiren a una inclusión genuina (Maggio, 2012), sus usos se despliegan y desarrollan de distintas maneras en las diferentes jurisdicciones, instituciones y espacios educativos. Tal como se observa en la Figura 2, en el período de aislamiento obligatorio, se capturan diversos escenarios, con el objetivo de enseñar y mantener de manera remota el vínculo y el encuentro pedagógico entre docentes y estudiantes.

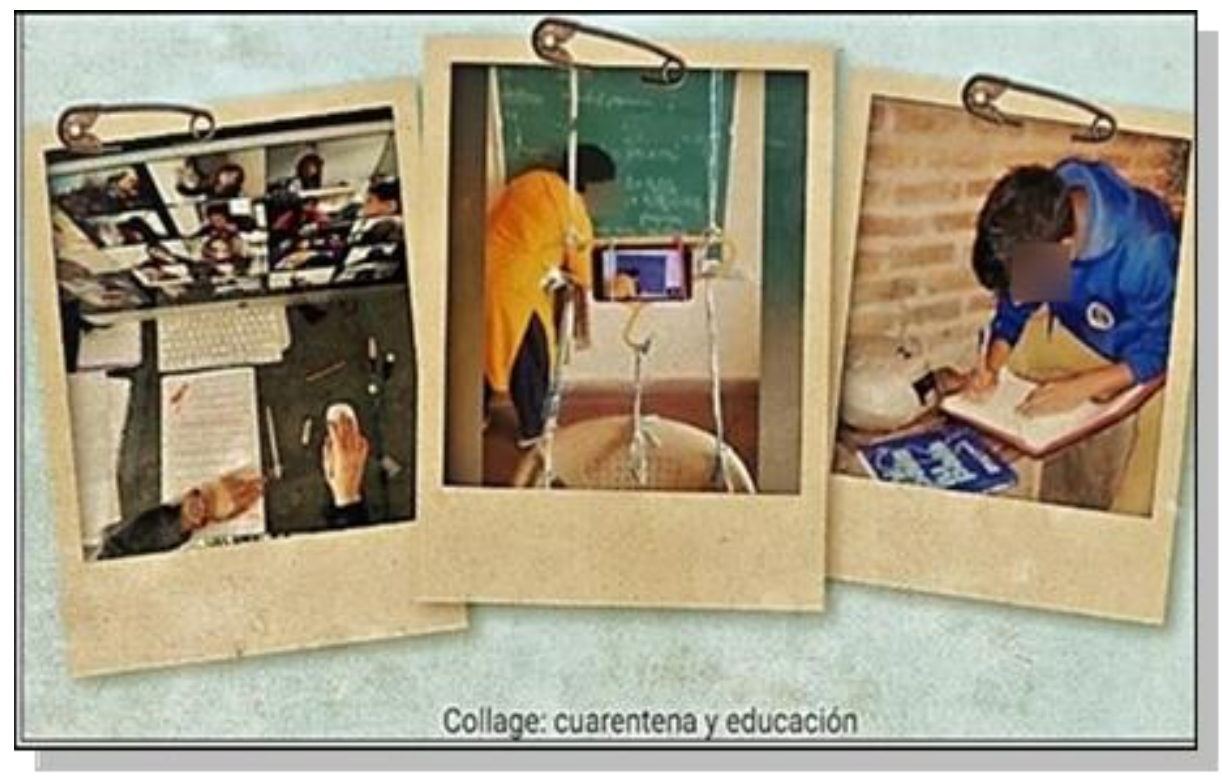

Fuente: "Collage de producción propia: educación y cuarentena". Imágenes tomadas de la red social Facebook y de Sepúlveda, P. (2 abril 2020). Educación en línea en cuarentena: ¿Cómo ser más que un docente que lee diapositivas y entusiasmar a los alumnos? La Tercera. Recuperado de https://cutt.ly/ssZ5LPJ

A la luz de estos hechos, surge la necesidad de reflexionar y aportar algunas ideas sobre el tema. En este trabajo intentaremos, en primer lugar y de forma breve, reconstruir un marco general respecto del impacto de la pandemia en las instituciones del ámbito educativo y describir la figura del Estado como actor clave. En segundo lugar, intentaremos recuperar argumentos para establecer la importancia y la necesidad de generar condiciones para favorecer la inclusión de las TIC de manera integral en las propuestas pedagógicas que sirvan de base para la continuidad 
pedagógica ante escenarios cambiantes -sobre todo aquellos que tienen la característica de ser inesperados. En tercer lugar, presentaremos algunas ideas en torno a la construcción de ciertos matices de escenarios institucionales posibles, a partir de la identificación de alteraciones que la pandemia expuso y, por último, plantearemos algunas reflexiones sobre lo trabajado, sobre escenarios de pospandemia y sobre acciones que contemplen una mirada hacia el futuro para el desarrollo de propuestas educativas alternativas en contexto y a largo plazo, pensando en varios futuros posibles.

\section{La alteración institucional frente a lo inesperado}

Las instituciones, en tanto expresión de normas, valores, creencias y mandatos de alcance universal, con formaciones sociohistóricas que nos anteceden, operan sobre nosotros/as como individuos, grupos y organizaciones, ordenando nuestra percepción y dirigiendo una serie de rasgos que se presentan como constantes y permiten generar la impresión de un "orden natural de las cosas" (Fernández, 1994). Sin embargo, esta pandemia alteró considerablemente esa normalidad u orden natural: nos impidió reunirnos físicamente a celebrar un evento social, nos impidió compartir un mate, implementamos otras formas de saludarnos, posiblemente alteró diversas planificaciones y también interrumpió la concurrencia a las instituciones educativas y la asistencia a clases de manera presencial.

En este escenario, la pandemia por coronavirus, de manera inédita y a una velocidad sin precedentes, nos interpeló y nos desafió a rediseñar, imaginar y proponer nuevas configuraciones o formas de actuar y de relacionarnos y, a su vez, abrió el debate sobre la necesidad de construir políticas públicas federales de distinta índole (aunque en este artículo, haremos hincapié en el ámbito educativo especialmente).

Amplificando la mirada, podemos analizar algunas cuestiones sobre el escenario educativo de América Latina y el Caribe. Para ello, resulta interesante recuperar una publicación realizada por miembros de la División de Educación del BID, del 9 de abril del 2020 en su blog "Enfoque Educación". Dicha publicación, en primera instancia, expuso que las comunidades educativas debieron tomar medidas que garantizaran:

- El vínculo estudiante-docente y familia-escuela.

- La entrega de contenido alineado al currículo escolar.

- El acompañamiento y monitoreo del proceso de aprendizaje.

En este sentido se señala que, con relación a las medidas adoptadas, la mayoría de los países optó por combinar diversos canales (como medios masivos, plataformas de aprendizaje, material digital e impreso), aunque en gran medida esta combinación estuvo supeditada a la disponibilidad de infraestructura y a los contenidos. En la siguiente tabla se puede observar gráficamente el esquema de las medidas adoptadas por distintos países de América Latina y El Caribe: 


\section{Tabla 1.}

\section{Medidas adoptadas por distintos países de América Latina y El Caribe}

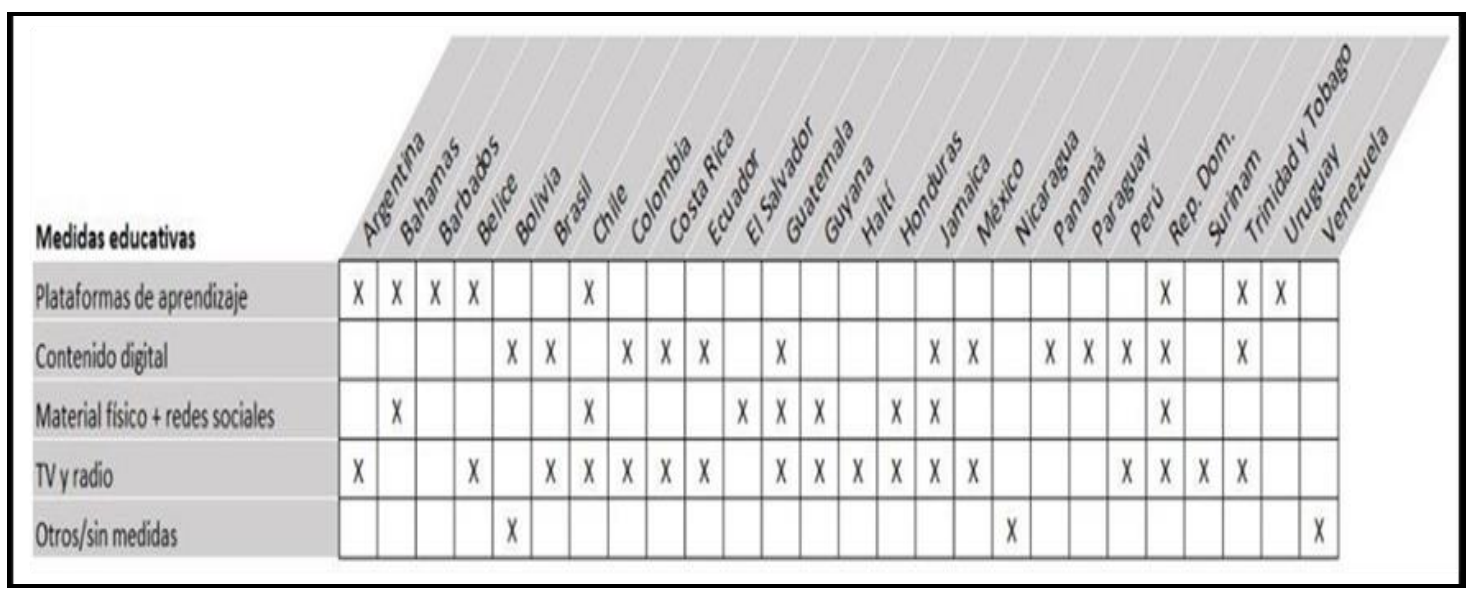

Fuente: tomado de Enfoque Educación, Blog de la División de Educación del BID. "Cierre de escuelas: el desafío que el COVID- 19 impuso a los sistemas educativos de ALC" (09/04/2020) Recuperado de https://blogs.iadb.org/educacion/es/cierredeescuelas/

En este marco se vislumbra que en algunos países el rol del Estado ha sido clave y ha jugado un papel fundamental, en tanto desplegó determinadas políticas públicas (no sólo en el ámbito educativo) destinadas a establecer consenso respecto de proteger el derecho a la educación.

En Argentina, particularmente, se apuntó a brindar (desde distintas ópticas) condiciones que sostuvieran el vínculo pedagógico y garantizaran la continuidad de los aprendizajes de los y las estudiantes. Aquí, sólo mencionaremos algunas de índole educativa destinadas a docentes y estudiantes de todo el país, con el objetivo de colaborar con las condiciones para la continuidad de las actividades de enseñanza y de aprendizaje en el sistema educativo nacional. A modo de ejemplo, se puede mencionar el portal Seguimos Educando del Ministerio de Educación de la Nación, o Continuemos Estudiandoii de la Dirección General de Cultura y Educación de la Provincia de Buenos Aires. No obstante, al mismo tiempo, por fuera del ámbito estatal, se han realizado un sinfín de conferencias, webinars, conversatorios, como también innumerables intercambios de experiencias y de recursos a través de múltiples plataformas que, en su mayoría, apuntaron a la colaboración, el acompañamiento y la reflexión sobre las prácticas pedagógicas en este contexto inesperado, transitorio y singular.

Por otra parte, si bien se puede afirmar que todas las provincias han dispuesto herramientas digitales educativas para dar respuesta a la continuidad pedagógica en la pandemia, estas acciones han tenido alcances y recursos dispares. Desde una mirada macro, se deja entrever la necesidad de volver a generar una política pública federal que contemple las distintas realidades y contextos. En esa línea, el Ministerio de Educación de la Nación avanza en el Plan Federal de Conectividad Juana Manso, que buscará integrar y brindar acceso a la tecnología los y las estudiantes de todo el país e incluirá la creación de una plataforma federal de enseñanza con acceso gratuito, con la posibilidad de brindar conectividad en los barrios más vulnerados. 
En sintonía con lo manifestado hasta aquí, pero haciendo "Zoom" en las instituciones educativas, éstas se vieron forzadas a adoptar medidas abruptas, urgentes, eficaces y posiblemente muy diferentes de las planificadas para el ciclo escolar o académico del año 2020. En variados planos, existió una alteración significativa de las dinámicas institucionales, sus procesos de producción, los procesos de enseñanza y de aprendizaje y, por supuesto, las prácticas de todos sus actores. La velocidad y la urgencia que requería tomar decisiones y medidas es un hecho que, en lo contemporáneo y en nuestra realidad cercana, no tuvo ni tiene precedentes. Sin embargo, subyace un aspecto más, la pandemia del COVID-19 dejó en evidencia lo que ya se venía gestando por fuera de las paredes de distintas organizaciones institucionales: la decadencia o la crisis de las instituciones de la modernidad y la necesidad de diseñar y recrear por fuera de los marcos caducos, que siguen formateando nuestras conductas e identidades.

Michel Serres en 2013 publica su libro Pulgarcita, en el que advierte que, sin darnos cuenta, en un intervalo breve de tiempo nació un nuevo humano que ya no tiene la misma cabeza que sus antepasados, ni habita el espacio de la misma manera. Este se comunica y percibe el mundo de otra manera. Sin embargo, frente a estas mutaciones las "instituciones relucen con un brillo semejante al de las constelaciones que, según nos enseñan los astrónomos, ya están muertas desde hace un largo tiempo" (2013, p. 32). En estas líneas, se deja entrever la idea y necesidad de imaginar instituciones elásticas, que respondan a problemáticas y necesidades específicas, a los cambios permanentes y las mutaciones de este tiempo.

Creemos en la necesidad de plantear nuevas formas de institucionalización que se encuentren en línea con los cambios sociales y culturales (y que también respondan a sucesos repentinos como el que transitamos con la pandemia). Nos preguntamos ¿cómo imaginamos el futuro en esta nueva normalidad? ¿cómo las TIC podrían posibilitar que nuestras instituciones adquieran más dinamismo? ¿cómo nuestras ideas se transforman en acciones?

\section{Instituciones atravesadas por las Tecnologías de la Información y Comunicación}

Las tendencias culturales de la contemporaneidad penetraban las paredes de las instituciones educativas, sin embargo, en su interior, estaban siendo resistidas y no lograban descomponer el status quo. A partir de la suspensión de clases presenciales debido a la pandemia ¿algo sucedió? Si bien en este momento no es posible llegar a una conclusión definitiva, se puede advertir que algunas de esas marcas cobraron impulso. En este sentido, se reflejan mecanismos de colaboración a través de variadas plataformas, múltiples redes sociales, medios de distinta índole. Sin embargo, esto ha sido posible en la medida en que, por un lado, se trastocaron las prácticas diarias y, por otro lado, en función de la disponibilidad de los recursos (dispositivos, conexión a internet, adquisición de software, etc.).

En este marco, volvemos a identificar que resulta relevante fortalecer las políticas de inclusión digital, en tanto podrían reducir algunas brechas aún vigentes y podrían mejorar los procesos de enseñanza y aprendizaje en las instituciones educativas, considerando que en la sociedad del conocimiento, el uso y la apropiación de las Tecnologías de Información y Comunicación son un factor clave para alcanzar resultados estratégicos y actualmente adquieren mayor significatividad como respuesta traducida en acciones concretas para hacer frente a determinados sucesos 
inesperados -como el que desembocó la pandemia del coronavirus- que incluye la suspensión de clases presenciales a nivel nacional.

Las experiencias vividas en este contexto en torno a la utilización de las TIC para sostener la continuidad pedagógica -tal como lo señalamos anteriormenteestuvieron condicionadas por la disponibilidad de recursos y las dinámicas institucionales particulares. En casi todos los casos, se trataron de experiencias de enseñanza y de aprendizaje remoto en situación de emergencia. Con esto queremos decir que, si bien la pandemia visibiliza que existen deudas y brechas por saldar, la mayoría de las instituciones educativas están en camino hacia el desarrollo y la implementación de recursos y herramientas digitales.

En este sentido, se hace evidente la necesidad de crear y repensar

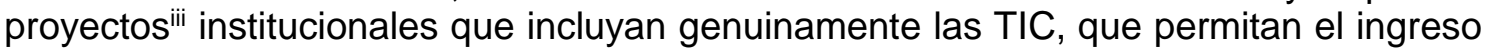
de lo digital, más allá del contexto de emergencia ¿y que implicaría pensar en proyectos a largo plazo? Desde una perspectiva compleja, significa reconocer e identificar la dimensión tecnológica y las marcas de la contemporaneidad consustancial a todo conocimiento. Para ello es necesario, vincular desde un enfoque sistémico, medios, métodos, instrumentos, técnicas y procesos en vista a analizar los problemas y administrar soluciones (De Pablos Pons, 2009).

Mientras que las TIC son la herramienta por definición en los entornos de alta disposición tecnológica, en los terrenos donde la conectividad es escasa o nula, se recurrieron a acciones que utilizan medios de comunicación masiva, como la radio o los cuadernillos en soporte papel. Estas configuraciones son dispares y diferentes dado que los escenarios, las condiciones de formación y las situaciones contextuales son desiguales a lo largo y ancho de nuestro país (y en el mundo en general). Aquí vuelve a aparecer la necesidad de una política federal de inclusión educativa digital, que considere las necesidades diversas e integre a la totalidad de las provincias de nuestro país en el desarrollo de un proyecto nacional que abarque estrategias pedagógicas integradas apoyadas en una ecología de plataformas educativas (AxE, 2020).

\section{Matices de escenarios y posibilidades de acción}

En este apartado, nos centraremos en un plano singular y realizaremos algunos señalamientos sobre las instituciones educativas. Para ello hemos decidido hablar de escenarios, apoyados en tres ideas fuerza. Por un lado, la definición de escenario, como lugar donde ocurre o se desarrolla un suceso o un conjunto de circunstancias que rodean a una persona (o colectivo). Por otro lado, teniendo en cuenta a los estudios de prospectiva donde, el método de escenarios es muy utilizado debido a que permite cuestionar y pensar creativamente acerca del futuro y pensar en múltiples futuros posibles, más allá del futuro tendencial y, por último, el uso de la idea de matiz, que da lugar a la posibilidad de identificar diferentes momentos que se plantean en pos de sostener y fortalecer determinadas dinámicas.

Consideramos que estas nociones nos abren la puerta para poder dialogar sobre diferentes experiencias educativas originadas en respuesta a la crisis, consecuencia de la pandemia, y que se apoyaron en las TIC como medio y entorno. Particularmente aquí enfocamos la mirada hacia expresiones que se desarrollaron en instituciones educativas en tanto entidades singulares, que se podrían agrupar atendiendo a algunos rasgos comunes. 
Partiendo del análisis del despliegue de distintas acciones para la continuidad pedagógica y el sostenimiento del vínculo pedagógico, consideramos que la contingencia ha dado lugar a la construcción de tres tipos posibles de escenarios institucionales que enfrentan (y enfrentarán) desafíos diferentes en torno a proyectos integrales de inclusión de las TIC en las instituciones educativas. Si bien imaginamos éstos, también se podrían pensar en otros matices, porque nada es acabado ni definitivo, incluso se contempla la fluidez, como característica que comprende el desplazamiento y los cambios con facilidad:

a) Escenarios institucionales acelerados. Utilizamos el término acelerados para referirnos a aquellos escenarios institucionales que no tenían desarrollado ningún tipo de proyecto de inclusión de TIC pero que, ante la contingencia y la imperante enseñanza remota tuvieron que comenzar a idear, delinear y diseñar propuestas que incluyeran acciones destinadas a crear las bases de proyectos que contemplen las TIC, en forma de experimentación para el sostenimiento de la continuidad pedagógica.

b) Escenarios institucionales dinámicos. Aquí se incluyen escenarios institucionales que quizás venían desarrollando algún tipo de proyecto institucional que incluía herramientas tecnológicas digitales y TIC en propuestas pedagógicas, en tanto reconocen el valor de las tecnologías como rasgo de época, que constituyen un entorno que enriquece y transforma nuestras experiencias. Si bien se vieron alterados y tuvieron que ser hackeados para redefinir y acomodar las acciones a las distintas realidades de los actores en un escenario de contingencia, contaban con experiencias previas (dinámicas) que facilitaron un reacomodamiento con mayor agilidad a los cambios inesperados. Al mismo tiempo, estas instituciones poseen capitales (humanos y económicos) que posibilitan el empleo de tecnologías en las prácticas de enseñanza y de aprendizaje.

c) Escenarios institucionales comprometidos. Se vislumbran escenarios institucionales que, a pesar de reconocer que las tecnologías (la digital y las TIC, en particular) están cada vez más presentes en nuestra cotidianidad, que modifican nuestros hábitos y formas de relacionarnos, son escenarios institucionales en los que no se contó con los recursos y/o medios necesarios para desplegar algún tipo de proyecto o bien, existieron -y existen- otro tipo de necesidades ante un escenario de emergencia -como el de la pandemia- (o ambas combinaciones). En general, tienen la característica de poseer poca o nula conectividad, no tienen acceso a dispositivos -o es más bien restringido- o podrían ser espacios educativos con características específicas.

En este punto nos interesa destacar que, cada institución, se ubica en un contexto histórico, cultural y social particular, forja tradiciones, lógicas y prácticas que la hacen única e irrepetible. No obstante, estos tipos de escenarios institucionales acelerados, dinámicos y comprometidos- forman parte de un escenario más amplio, que contempla espacios educativos contextualizados, y que podríamos llamar escenarios líquidos -siguiendo la idea de Bauman (2003)- porque se tratan de escenarios que comprenden cambios constantes y se encuentra en estado de transitoriedad. Además, están cercados por distintos factores y no conservan su forma constantemente.

Que un escenario tenga la característica de ser líquido no implica que en él no se puedan generar acciones colectivas a modo de esquema de trabajo. En palabras de Maggio, se debería pensar en las escuelas como motores de producción y de ideas originales, que generen soluciones para las comunidades, estrategias de acción e intervención y nuevas formas de generar redes (Proyecto Educar 2050, 
2019). Si hay una lección de aprendizaje que la pandemia nos está dejando, es la necesidad de poder pensar y construir en redes y de forma colaborativa. Un claro ejemplo -del plano de la salud y de la investigación- es la contribución y colaboración de distintas universidades para el desarrollo conjunto de una vacuna para la cura del COVID-19iv.

En sintonía con lo planteado, cabe señalar que la red o las redes, se han convertido en la forma organizativa más eficiente en tanto que posee rasgos como la flexibilidad, la adaptabilidad, la capacidad de supervivencia y son altamente moldeables por las fuerzas sociales, la cultura, la política y las estrategias económicas (Castells, 2012). La sociedad contemporánea opera bajo esta lógica, sin embargo, la red o las redes funcionan sobre la lógica de inclusión- exclusión en tanto que no todas las personas participan en y las redes, aunque su fuerza alcance a todo y todos/as

Sabemos que las tecnologías no son neutrales, responden a determinados preceptos, que pueden generar y amplificar formas de poder y control por lo que se necesita comprender lo mejor posible esta naturaleza. Preguntarse cómo y para qué usar la tecnología es un aspecto de naturaleza política que, quienes diseñan e implementan proyectos que promueven la inclusión de tecnologías digitales y de las TIC, deberán formularse en tanto que tiene implicancias y efectos en las experiencias de enseñanza, de aprendizaje y en las relaciones humanas.

\section{Lo que la pandemia nos deja para pensar la pospandemia}

A lo largo de este trabajo, intentamos recuperar algunas marcas y efectos que en este tiempo la pandemia está produciendo en nosotros/as como sujetos sociales, aunque, particularmente, acentuamos la mirada en el impacto sobre las instituciones educativas.

En este sentido como primera instancia, destacamos el papel protagónico del Estado en clave de diseñar y llevar a cabo políticas de índole federal que apuntan a ofrecer garantías de accesibilidad, lo más universales, justas y sustentables posibles. Existe un consenso en torno a los niveles de desigualdad respecto de los alcances y los recursos, por tanto, las acciones deben estar orientadas a reducir esta brecha.

En otra línea recuperamos la necesidad de generar proyectos institucionales que incluyan TIC en clave integral y a largo plazo, entendiendo que ninguna tecnología puede contribuir a desarrollar por sí sola el conjunto de capacidades humanas, sino que se expresa a partir de una inclusión genuina (Maggio, 2012) que reconoce el lugar y el sentido de la tecnología en la construcción del conocimiento y se plasma en el diseño de propuestas pedagógicas. Al mismo tiempo, se reconoce que las tecnologías no son neutrales, sino que responden a determinados fines y que debemos conocer y reflexionar sobre las consecuencias de su utilización.

Por otro lado, analizamos que la singularidad de la emergencia dejó entrever algunos matices de escenarios institucionales. Si bien es posible advertir que la pandemia implicó una alteración inesperada, cada escenario debió ensayar alternativas, redefinir sus planificaciones y modalidades de trabajo. Aquí radica la singularidad. Sin embargo, este suceso nos ofrece algunos elementos para conjugar esa singularidad en construcciones más amplias para el escenario pospandemia, por ejemplo: 
- Preguntarnos cómo las instituciones tuvieron que reconstruirse rápidamente, conjugando comunidades y redes y si pueden dar cuenta de ese proceso para registrarlo y trabajar desde allí.

- Comprender que somos las personas las que podemos transformar y cambiar los contextos o los ámbitos que habitamos. Se tratará de expandir no sólo la universidad o la escuela sino además las acciones, las redes, el trabajo con otros/as. Se trata de generar comunidad y construir redes de acción conjuntas para observar el complejo entramado de múltiples conocimientos, afrontar críticamente las lógicas corporativas y asumir como desafío los diversos e inesperados caminos que se abren mediante la apropiación de las TIC por los y las actores involucrados (Benítez Larghi, 2020).

- Considerar la necesidad de pensar en proyectos a largo plazo, que se desplieguen en instituciones transformadas, que contemplen la fluidez de los cambios y la porosidad de los escenarios. Lo inesperado como factor común a lo institucional, que transforma identidades y relaciones.

- Repensar las relaciones, ahora mediatizadas de forma remota, entre actores educativos. Al mutar la forma de enseñar y de aprender surgen nuevas formas de relacionarnos ¿cómo analizamos y abordamos lo que en ellas acontece? ¿esta nueva normalidad dará lugar a la instalación de una educación híbrida en los diferentes niveles del sistema educativo? ¿cuáles son las condiciones y garantías para su desarrollo? ¿la normativa vigente es suficiente? ¿abarca a todas las instituciones y actores del sistema?

Los problemas complejos nunca son definitivos, y el cambio a lo largo del proceso de transformación, es la constante. Se percibe necesaria una mirada de futuro para pensar cualquier proyecto que permita cuestionar y pensar de forma creativa, flexible e inclusive pensar en múltiples futuros posibles, dando lugar y acompañando la incertidumbre.

\section{Referencias bibliográficas}

Bauman, Z. (2003). Modernidad líquida. Buenos Aires, Argentina: Fondo de Cultura Económica.

Benítez Larghi, S. (2020). Desafíos de la inclusión digital en Argentina. Una mirada sobre el Programa Conectar Igualdad. Revista de Ciencias Sociales, 33 (46), 131-154. Recuperado

http://www.scielo.edu.uy/scielo.php?script=sci arttext\&pid=S0797$\underline{55382020000100131 \& \operatorname{lng}=\mathrm{es} \& \mathrm{nrm}=\text { iso }}$

Boletín Oficial de la República Argentina (2020). Decreto 297/2020. Ciudad de Buenos Aires, Argentina.

Castells, M. (2012). Comunicación y poder. México: Siglo XXI.

De Pablos Pons, J. (2009). Tecnología educativa. La formación del profesorado en la era de internet. Málaga, España: Ediciones Aljibe. 
Fernández, L. (1994). Instituciones educativas. Dinámicas institucionales en situaciones críticas. Buenos Aires, Argentina: Paidós.

Hodges, C., Moore, S., Lockee, B., Torrey, T. \& Bond, A. (2020). The Difference Between Emergency Remote Teaching and Online Learning. [EDUCAUSE]. Recuperado de https://er.educause.edu/articles/2020/3/the-differencebetween-emergency-remote-teaching-and-online-learning

INTEGRA (2007). Herramientas para la gestión de proyectos educativos con TIC. UNESCO. Sede Regional Buenos Aires, Argentina. Recuperado de https://unesdoc.unesco.org/ark:/48223/pf0000158068

Libedinsky, M. (2016). La innovación educativa en la era digital. Ciudad Autónoma de Buenos Aires, Argentina: Paidós.

Maggio, M. (2012) Enriquecer la Enseñanza. Los ambientes con alta disposición tecnológica como oportunidad. Buenos Aires, Argentina: Paidós.

Manso, M., Pérez, P., Libedinsky, M., Light, D., y Garzón, M. (2011) Las TIC en las aulas. Experiencias latinoamericanas. Buenos Aires, Argentina: Paidós.

Ministerio de Educación. Boletín Oficial de la República Argentina. Resolución 106/2020. Recuperado https://www.boletinoficial.gob.ar/detalleAviso/primera/226751/20200316

Ministerio de Educación de la Nación Argentina (2020). El Ministerio de Educación de la Nación avanza en el Plan Federal de Conectividad. Recuperado de https://www.argentina.gob.ar/noticias/el-ministerio-de-educacion-de-la-nacionavanza-en-el-plan-federal-de-conectividad

Proyecto Educar 2050. Mediar: Mesas de Diálogo para el Aprendizaje en Argentina (2019). Ciudad Autónoma de Buenos Aires, Argentina. Recuperado de https://educar2050.org.ar/documento-mediar-web/

Observatorio Argentinos por la Educación [AxE] (2020). Herramientas digitales educativas provinciales. Recuperado de https://cms.argentinosporlaeducacion.org/media/reports/Herramientas provinc iales educativas.pdf

Pardo Kuklinski, H., y Cobo, C. (2020). Expandir la universidad más allá de la enseñanza remota de emergencia. Ideas hacia un modelo híbrido postpandemia. Barcelona, España: Outliers School. Recuperado de https://outliersschool.net/la-universidadpostpandemia-pardo-kuklinski-cobo/

Pineda Acero, J. (2016). Diseño de proyectos educativos mediados por TIC: un marco de referencia. Opción, 32 (10), 479-499. Recuperado de https://www.redalyc.org/pdf/310/31048901026.pdf

Preciado, P. (2020). Aprendiendo del Virus. El País. 163- 185. Recuperado de https://sxpolitics.org/es/wp-content/uploads/sites/3/2020/04/Sopa-de-WuhanASPO.pdf.pdf-pa\%CC\%81ginas-163-185.pdf 
Serres, M. (2013). Pulgarcita: el mundo cambió tanto que los jóvenes deben reinventar todo: una manera de vivir juntos, instituciones, una manera de ser y conocer. Buenos Aires, Argentina: Fondo de Cultura Económica.

Vásquez, M., Ortiz, M., Álvarez Marinelli, H., Pérez Alfaro, M., Arias Ortiz, E., y Bergamaschi, A. (2020). Cierre de escuelas: el desafío que el COVID-19 impuso a los sistemas educativos de ALC. [Enfoque Educación] Recuperado de https://blogs.iadb.org/educacion/es/cierredeescuelas/

\section{Notas}

' EDUCAR es una Sociedad del Estado (SE) responsable por el portal educativo oficial del Ministerio de Educación de la República Argentina.

ii Continuemos Estudiando es una plataforma que ofrece materiales, recursos y propuestas de actividades, organizadas y sugeridas desde las direcciones de los niveles y modalidades de la Provincia de Buenos Aires.

iii Para indagar sobre características de proyectos que llevan adelante dos universidades públicas argentinas en materia de desarrollo de una vacuna contra el COVID-19, sugerimos consultar en https://www.redaccion.com.ar/vacuna-argentinacontra-el-covid-19-las-caracteristicas-de-los-proyectos-que-lideran-dos-universidadespublicas/

iv Proyectos en el sentido de organización y estructura para alcanzar un objetivo concreto. 